\title{
Application of Daftardar Jafari Method to First Grade MHD Squeezing Fluid Flow in a Porous Medium with Slip Boundary Condition
}

\author{
Inayat Ullah, M. T. Rahim, and Hamid Khan \\ Department of Mathematics, National University of Computer and Emerging Sciences, FAST, Peshawar Campus, \\ Peshawar 25000, Pakistan
}

Correspondence should be addressed to Inayat Ullah; p119952@nu.edu.pk

Received 25 March 2014; Accepted 2 June 2014; Published 18 June 2014

Academic Editor: Hossein Jafari

Copyright (c) 2014 Inayat Ullah et al. This is an open access article distributed under the Creative Commons Attribution License, which permits unrestricted use, distribution, and reproduction in any medium, provided the original work is properly cited.

In the present work, in the presence of magnetic field and with slip boundary condition, squeezing flow of a Newtonian fluid in a porous medium between two large parallel plates is investigated. The governing equations are transformed to a single nonlinear boundary value problem. Daftardar Jafari Method (DJM) is used to solve the problem in order to obtain the velocity profile of the fluid. By using residual of the problem, the validity of solution is established. The velocity profile is argued through graphs for various values of parameters.

\section{Introduction}

Movable boundary stressed by vertical velocity causes squeezing flow [1]. The most practical examples of squeezing flows are polymer processing, compression, and injection modeling. The preliminary results in this field are carried out by Stefan [2]. The Newtonian squeezing fluid flow between two parallel plates is studied in [3]. The study of thin Newtonian liquid films squeezed between two plates is investigated by Grimm (Figure 1) [4]. Squeezing flow, under the influence of magnetic field, has many applications in the field of chemical engineering and is experimented by many researchers [5-8].

Molecules near the boundary show slip at the boundary when the weight of molecule is high. In case of thin film problems, rarefied fluid problems, fluids containing concentrated suspensions, and flow on multiple interfaces, no-slip boundary condition does not work. The general boundary condition, which shows the fluid slip at the surface, is introduced by Navier [9]. The difference of the velocities of fluid and of the boundary, according to Navier, is proportional to the shear stress at that boundary. This proportionality constant is called slip parameter.
The slip condition has its significance when fluids with elastic character are considered [10]. In medical sciences, particularly in polishing artificial heart valves and internal cavities, the slip condition is highly considered [11]. The effect of slip condition on MHD squeezing fluid flow is discussed in [12]. For rotating flow of a third grade fluid in a nonporous medium, the effects of slip condition are investigated by Hayat and Abelman [13]. The same model with the extension of porous medium is argued by Abelman et al. [14]. Newtonian fluid was considered by Ebaid [15] to study the effects of magnetic field and wall slip conditions on the peristaltic transport in an asymmetric channel.

There are many applications of magnetohydrodynamics (MHD) of an electrically conducting fluid in geophysics, astrophysics, engineering, and other industrial areas. More to the point, the flows of electrically conducting fluid through porous medium have acquired exceptional rank and have been the spotlight of interest of many researchers. The precise applications are initiated in the study of ground water flow, irrigation problems, crude petroleum recovery, heat-storage beds, thermal and insulating engineering, chromatograph, and chemical catalytic reactors. 
TABLE 1: Absolute residuals for different values of $\gamma$ keeping Me and Re fixed.

\begin{tabular}{|c|c|c|c|c|c|}
\hline \multirow{2}{*}{$z$} & \multicolumn{5}{|c|}{ Absolute residuals for different values of slip parameter } \\
\hline & $(\gamma=0.2)$ & $(\gamma=0.4)$ & $(\gamma=0.6)$ & $(\gamma=0.8)$ & $(\gamma=1.0)$ \\
\hline 0. & 0. & 0. & 0. & 0. & 0. \\
\hline 0.1 & $4.15 \times 10^{-10}$ & $2.73 \times 10^{-11}$ & $7.69 \times 10^{-15}$ & $7.60 \times 10^{-14}$ & $8.65 \times 10^{-14}$ \\
\hline 0.2 & $2.00 \times 10^{-7}$ & $1.12 \times 10^{-8}$ & $2.59 \times 10^{-12}$ & $4.12 \times 10^{-11}$ & $4.60 \times 10^{-11}$ \\
\hline 0.3 & $7.04 \times 10^{-6}$ & $1.97 \times 10^{-7}$ & $2.27 \times 10^{-10}$ & $1.74 \times 10^{-9}$ & $2.00 \times 10^{-9}$ \\
\hline 0.4 & $8.48 \times 10^{-5}$ & $3.31 \times 10^{-6}$ & $1.55 \times 10^{-8}$ & $2.66 \times 10^{-8}$ & $2.70 \times 10^{-8}$ \\
\hline 0.5 & $5.87 \times 10^{-4}$ & $1.03 \times 10^{-4}$ & $3.90 \times 10^{-7}$ & $2.35 \times 10^{-7}$ & $2.20 \times 10^{-7}$ \\
\hline 0.6 & $3.04 \times 10^{-3}$ & $1.21 \times 10^{-3}$ & $3.33 \times 10^{-6}$ & $1.50 \times 10^{-6}$ & $1.30 \times 10^{-6}$ \\
\hline 0.7 & $1.38 \times 10^{-2}$ & $9.17 \times 10^{-3}$ & $2.40 \times 10^{-5}$ & $7.66 \times 10^{-6}$ & $6.10 \times 10^{-6}$ \\
\hline 0.8 & $5.79 \times 10^{-2}$ & $5.27 \times 10^{-2}$ & $1.30 \times 10^{-4}$ & $3.40 \times 10^{-5}$ & $2.43 \times 10^{-5}$ \\
\hline 0.9 & $2.26 \times 10^{-1}$ & $2.48 \times 10^{-1}$ & $5.50 \times 10^{-4}$ & $1.30 \times 10^{-4}$ & $8.60 \times 10^{-5}$ \\
\hline 1. & $8.02 \times 10^{-1}$ & $9.96 \times 10^{-1}$ & $2.00 \times 10^{-3}$ & $5.00 \times 10^{-4}$ & $3.00 \times 10^{-4}$ \\
\hline
\end{tabular}

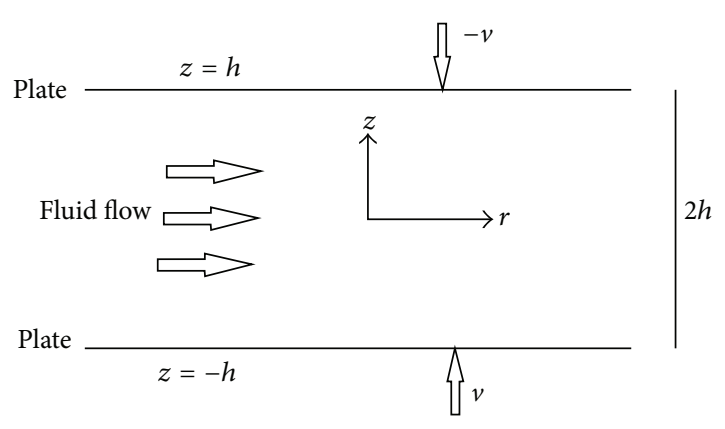

Figure 1: Squeezing flow of Newtonian fluid between two large parallel plates.

The idea of DJM is introduced by Daftardar-Gejji and Jafari $[16,17]$. This scheme (DJM) is computer-friendly and effective to deal with highly nonlinear boundary value problems [18]. The convergence of DJM can be found in [19] where fractional boundary value problems with Dirichlet boundary conditions are considered. To compare different analytic solutions for squeezing fluid flow between two parallel plates, DJM is successfully used [20]. Using this scheme, excellent results are obtained in [21] for the solution of fifth- and sixth-order boundary value problems.

The objective of this research paper is to use DJM for studying, with slip boundary condition, first grade MHD squeezing fluid flow in a porous medium bounded by two parallel plates approaching each other slowly. The basic idea of the proposed scheme is discussed and the modeled boundary value problem is solved through it. The results along with residuals, for different values of parameters, are shown in Tables 1, 2, 3, 4, and 5 . The effects on velocity profile of the fluid, by the influence of different parameters, are shown in the graphs.

\section{Mathematical Model}

The Navier-Stokes equations for such fluid flow between two large parallel plates separated by $2 h$ distance are

$$
\begin{gathered}
\operatorname{div} \mathbf{v}=0 \\
\rho\left[\nabla\left(\frac{1}{2} \mathbf{v}^{2}\right)-\mathbf{v} \times \omega\right]=-\nabla p+\mu(-\nabla \times \omega)-\left(\frac{\mu}{k}+\eta B^{2}\right) \mathbf{v},
\end{gathered}
$$

where $\rho$ denotes the constant density, $\mu$ is viscosity, $\mathbf{v}$ is the velocity of plates, $\nabla$ denotes the material time derivative, $p$ denotes the pressure, $k$ is permeability, $B$ is the total magnetic field given by $B=B_{0}+b$, and $\eta$ denotes the electrical conductivity while $\omega$ is given by

$$
\omega=\nabla \times \mathbf{v}
$$

In the total magnetic field $B, B_{0}$ and $b$ are imposed and induced magnetic fields respectively. Consider $b$ as negligible as compared to $B_{0}, \rho, \mu$, and $\eta$ constant and $\mathbf{B}$ is perpendicular to $\mathbf{v}$ with no electric field in the fluid flow region; the involved magnetohydrodynamic force can be written as

$$
\mathbf{J} \times \mathbf{B}=-\eta B_{0}^{2} \mathbf{v}
$$

Here $J$ is the electric current density. Magnetic field is applied along the $z$-axis. In cylindrical coordinates, the components of the velocity $\mathbf{v}$ are given by $\mathbf{v}=\left(v_{r}, 0, v_{z}\right)$. If $v_{r}^{2}+v_{z}^{2}=u$, then by comparison we get;

$r$-component:

$$
\rho\left(\frac{\partial u}{\partial r}-\Omega v_{z}\right)+\frac{\partial p}{\partial r}=-\left(\mu \frac{\partial \Omega}{\partial z}+A v_{r}\right)
$$

$z$-component:

$$
\rho\left(\frac{\partial u}{\partial z}+\Omega v_{r}\right)+\frac{\partial p}{\partial z}=-\left(-\frac{\mu}{r} \frac{\partial(r \Omega)}{\partial r}+A v_{z}\right),
$$

where

$$
A=\frac{\mu}{k}+\eta B_{0}^{2}, \quad \Omega(r, z)=\frac{\partial v_{z}}{\partial r}-\frac{\partial v_{r}}{\partial z} .
$$


TABLE 2: Absolute residuals for different values of Re keeping Me and $\gamma$ fixed.

\begin{tabular}{|c|c|c|c|c|c|}
\hline \multirow{2}{*}{$z$} & \multicolumn{5}{|c|}{ Absolute residuals for different values of Reynolds number } \\
\hline & $(\operatorname{Re}=1.5)$ & $(\operatorname{Re}=2)$ & $(\mathrm{Re}=3.5)$ & $(\operatorname{Re}=4.0)$ & $(\operatorname{Re}=5.0)$ \\
\hline 0. & 0. & 0. & 0. & 0. & 0. \\
\hline 0.1 & $8.72 \times 10^{-14}$ & $1.63 \times 10^{-12}$ & $7.04 \times 10^{-11}$ & $1.44 \times 10^{-10}$ & $4.40 \times 10^{-10}$ \\
\hline 0.2 & $4.53 \times 10^{-11}$ & $9.03 \times 10^{-10}$ & $3.92 \times 10^{-8}$ & $8.03 \times 10^{-8}$ & $2.45 \times 10^{-7}$ \\
\hline 0.3 & $1.98 \times 10^{-9}$ & $3.82 \times 10^{-8}$ & $1.70 \times 10^{-6}$ & $3.52 \times 10^{-6}$ & $1.10 \times 10^{-5}$ \\
\hline 0.4 & $3.67 \times 10^{-8}$ & $5.52 \times 10^{-7}$ & $2.62 \times 10^{-5}$ & $5.47 \times 10^{-5}$ & $1.75 \times 10^{-4}$ \\
\hline 0.5 & $4.50 \times 10^{-7}$ & $4.24 \times 10^{-6}$ & $2.27 \times 10^{-4}$ & $4.83 \times 10^{-4}$ & $1.60 \times 10^{-3}$ \\
\hline 0.6 & $4.05 \times 10^{-6}$ & $2.04 \times 10^{-5}$ & $1.40 \times 10^{-3}$ & $2.00 \times 10^{-3}$ & $1.01 \times 10^{-2}$ \\
\hline 0.7 & $3.00 \times 10^{-5}$ & $6.20 \times 10^{-5}$ & $6.44 \times 10^{-3}$ & $1.43 \times 10^{-2}$ & $5.03 \times 10^{-2}$ \\
\hline 0.8 & $1.60 \times 10^{-4}$ & $7.30 \times 10^{-5}$ & $2.52 \times 10^{-2}$ & $6.00 \times 10^{-2}$ & $2.12 \times 10^{-1}$ \\
\hline 0.9 & $7.31 \times 10^{-4}$ & $4.40 \times 10^{-4}$ & $8.62 \times 10^{-2}$ & $2.05 \times 10^{-1}$ & $8.00 \times 10^{-1}$ \\
\hline 1. & $3.00 \times 10^{-3}$ & $4.00 \times 10^{-3}$ & $3.00 \times 10^{-1}$ & $7.00 \times 10^{-1}$ & $3.00 \times 10^{0}$ \\
\hline
\end{tabular}

TABLE 3: Absolute residuals for different values of Me keeping Re and $\gamma$ fixed.

\begin{tabular}{|c|c|c|c|c|c|}
\hline \multirow{2}{*}{$z$} & \multicolumn{5}{|c|}{ Absolute residuals for different values of Hartmann number } \\
\hline & $(\mathrm{Me}=1.5)$ & $(\mathrm{Me}=2)$ & $(\mathrm{Me}=3)$ & $(\mathrm{Me}=4.0)$ & $(\mathrm{Me}=5.0)$ \\
\hline 0. & 0. & 0. & 0. & 0. & 0. \\
\hline 0.1 & $1.30 \times 10^{-14}$ & $1.40 \times 10^{-14}$ & $2.00 \times 10^{-12}$ & $1.43 \times 10^{-10}$ & $1.20 \times 10^{-9}$ \\
\hline 0.2 & $6.40 \times 10^{-12}$ & $6.55 \times 10^{-12}$ & $9.32 \times 10^{-10}$ & $7.30 \times 10^{-8}$ & $6.14 \times 10^{-7}$ \\
\hline 0.3 & $3.00 \times 10^{-10}$ & $2.30 \times 10^{-10}$ & $3.50 \times 10^{-8}$ & $3.00 \times 10^{-6}$ & $2.40 \times 10^{-5}$ \\
\hline 0.4 & $6.00 \times 10^{-9}$ & $3.00 \times 10^{-9}$ & $4.44 \times 10^{-7}$ & $4.00 \times 10^{-5}$ & $3.13 \times 10^{-4}$ \\
\hline 0.5 & $8.00 \times 10^{-8}$ & $2.30 \times 10^{-8}$ & $3.20 \times 10^{-6}$ & $3.00 \times 10^{-4}$ & $2.32 \times 10^{-3}$ \\
\hline 0.6 & $8.00 \times 10^{-7}$ & $1.45 \times 10^{-7}$ & $1.60 \times 10^{-5}$ & $1.40 \times 10^{-3}$ & $1.20 \times 10^{-2}$ \\
\hline 0.7 & $5.51 \times 10^{-6}$ & $7.60 \times 10^{-7}$ & $6.20 \times 10^{-5}$ & $5.31 \times 10^{-3}$ & $5.00 \times 10^{-2}$ \\
\hline 0.8 & $3.13 \times 10^{-5}$ & $3.10 \times 10^{-6}$ & $2.12 \times 10^{-4}$ & $2.00 \times 10^{-2}$ & $1.55 \times 10^{-1}$ \\
\hline 0.9 & $1.50 \times 10^{-4}$ & $9.00 \times 10^{-6}$ & $7.00 \times 10^{-4}$ & $4.00 \times 10^{-2}$ & $4.40 \times 10^{-1}$ \\
\hline 1. & $6.00 \times 10^{-4}$ & $1.10 \times 10^{-5}$ & $2.20 \times 10^{-3}$ & $1.10 \times 10^{-1}$ & $1.10 \times 10^{0}$ \\
\hline
\end{tabular}

Introducing stream function $\psi(r, z)[12]$ and the generalized pressure $\widehat{p}=\rho u+p,(4)$ and (5) are reduced to

$$
\begin{gathered}
\frac{\partial \widehat{p}}{\partial r}-\frac{\rho}{r^{2}} \frac{\partial \psi}{\partial r} E^{2} \psi=\frac{\mu}{r} \frac{\partial}{\partial z} E^{2} \psi-\frac{A}{r} \frac{\partial \psi}{\partial z} \\
\frac{\partial \widehat{p}}{\partial r}-\frac{\rho}{r^{2}} \frac{\partial \psi}{\partial z} E^{2} \psi=-\frac{\mu}{r} \frac{\partial}{\partial r} E^{2} \psi+\frac{A}{r} \frac{\partial \psi}{\partial r},
\end{gathered}
$$

where

$$
E^{2}=\frac{\partial^{2}}{\partial r^{2}}-\frac{1}{r} \frac{\partial}{\partial r}+\frac{\partial^{2}}{\partial z^{2}} .
$$

Eliminating $\widehat{p}$ from (7) and using the transformation $\psi(r, z)=r^{2} y(z)$, we have

$$
y^{(i v)}(z)-\left(\frac{1}{k}+\frac{\eta B_{0}^{2}}{\mu}\right) y^{\prime \prime}(z)+2 \frac{\rho}{\mu} y(z) y^{\prime \prime \prime}(z)=0,
$$

with boundary conditions

$$
\begin{gathered}
y(0)=0, \quad y^{\prime \prime}(0)=0, \\
y(h)=\frac{v}{2}, \quad y^{\prime}(h)=\beta y^{\prime \prime}(h) .
\end{gathered}
$$

Using the nondimensional parameters

$$
\begin{gathered}
Y^{*}=\frac{y}{v / 2}, \quad z^{*}=\frac{z}{h}, \\
\operatorname{Re}=\frac{\rho h}{\mu / v}, \quad M e=h \sqrt{\frac{1}{k}+\frac{\eta B_{0}^{2}}{\mu}}
\end{gathered}
$$

and omitting the $*,(9)$ and (10) can be written as

$$
\begin{gathered}
Y^{(i v)}(z)-\operatorname{Me}^{2} Y^{\prime \prime}(z)+\operatorname{Re} Y(z) Y^{\prime \prime \prime}(z)=0, \\
Y(0)=0, \quad Y^{\prime \prime}(0)=0 \\
Y(1)=1, \quad Y^{\prime}(1)=\gamma Y^{\prime \prime}(1)
\end{gathered}
$$

with $\gamma=\beta / h$. Re and Me are Reynolds and Hartmann numbers, respectively.

\section{Daftardar Jafari Method}

For the function $y(z)=g(z)+L(y(z))+N(y(z))$, let $y(z)=\sum_{n=0}^{\infty} y_{n}(z)$ be the series solution. $g(z)$ denotes the source term and $L(y(z))$ and $N(y(z))$ are, respectively, linear 
TABLE 4: Absolute residuals for different values of Me and Re with $\operatorname{Re}>$ Me keeping $\gamma$ fixed.

\begin{tabular}{|c|c|c|c|c|c|}
\hline \multirow{3}{*}{$z$} & \multicolumn{5}{|c|}{ Residuals for different values of Hartmann and Reynolds numbers } \\
\hline & $\mathrm{Re}=2$ & $\operatorname{Re}=2.5$ & $\mathrm{Re}=4$ & $\operatorname{Re}=7$ & $\operatorname{Re}=10$ \\
\hline & $\mathrm{Me}=1.5$ & $\mathrm{Me}=2$ & $\mathrm{Me}=3$ & $\mathrm{Me}=5.0$ & $\mathrm{Me}=7.0$ \\
\hline 0 . & 0. & 0. & 0. & 0. & 0. \\
\hline 0.1 & $2.70 \times 10^{-12}$ & $7.00 \times 10^{-12}$ & $1.20 \times 10^{-11}$ & $4.00 \times 10^{-10}$ & $4.00 \times 10^{-10}$ \\
\hline 0.2 & $1.50 \times 10^{-9}$ & $3.64 \times 10^{-9}$ & $7.00 \times 10^{-9}$ & $2.00 \times 10^{-7}$ & $2.00 \times 10^{-7}$ \\
\hline 0.3 & $6.15 \times 10^{-8}$ & $1.53 \times 10^{-7}$ & $3.05 \times 10^{-7}$ & $8.00 \times 10^{-6}$ & $7.30 \times 10^{-6}$ \\
\hline 0.4 & $9.22 \times 10^{-7}$ & $2.30 \times 10^{-6}$ & $5.00 \times 10^{-6}$ & $1.05 \times 10^{-4}$ & $9.63 \times 10^{-5}$ \\
\hline 0.5 & $8.00 \times 10^{-6}$ & $2.00 \times 10^{-5}$ & $4.43 \times 10^{-5}$ & $8.04 \times 10^{-4}$ & $7.13 \times 10^{-4}$ \\
\hline 0.6 & $5.00 \times 10^{-5}$ & $1.14 \times 10^{-4}$ & $3.00 \times 10^{-4}$ & $4.32 \times 10^{-3}$ & $4.00 \times 10^{-3}$ \\
\hline 0.7 & $2.32 \times 10^{-4}$ & $5.21 \times 10^{-4}$ & $1.30 \times 10^{-3}$ & $2.00 \times 10^{-2}$ & $1.52 \times 10^{-2}$ \\
\hline 0.8 & $9.45 \times 10^{-4}$ & $2.00 \times 10^{-3}$ & $5.02 \times 10^{-3}$ & $6.40 \times 10^{-2}$ & $5.40 \times 10^{-2}$ \\
\hline 0.9 & $3.41 \times 10^{-3}$ & $7.00 \times 10^{-3}$ & $2.00 \times 10^{-2}$ & $2.00 \times 10^{-1}$ & $2.00 \times 10^{-1}$ \\
\hline 1. & $1.12 \times 10^{-2}$ & $2.00 \times 10^{-2}$ & $5.00 \times 10^{-2}$ & $6.00 \times 10^{-1}$ & $5.43 \times 10^{-1}$ \\
\hline
\end{tabular}

TABLE 5: Absolute residuals for different values of $\mathrm{Me}, \mathrm{Re}$, and $\gamma$ with $\mathrm{Re}<\mathrm{Me}$.

\begin{tabular}{|c|c|c|c|c|c|}
\hline \multirow{4}{*}{$z$} & \multicolumn{5}{|c|}{ Residuals for different values of $\gamma$ and Hartman and Reynolds numbers } \\
\hline & $\operatorname{Re}=2$ & $\operatorname{Re}=1.0$ & $\operatorname{Re}=3.5$ & $\operatorname{Re}=4.5$ & $\operatorname{Re}=5.0$ \\
\hline & $\mathrm{Me}=3$ & $\mathrm{Me}=1.5$ & $\mathrm{Me}=4$ & $\mathrm{Me}=6.0$ & $\mathrm{Me}=7.0$ \\
\hline & $\gamma=0.1$ & $\gamma=0.2$ & $\gamma=0.5$ & $\gamma=0.8$ & $\gamma=1.0$ \\
\hline 0. & 0. & 0. & 0. & 0. & 0. \\
\hline 0.1 & $9.42 \times 10^{-11}$ & $8.00 \times 10^{-12}$ & $1.22 \times 10^{-11}$ & $4.00 \times 10^{-11}$ & $4.00 \times 10^{-10}$ \\
\hline 0.2 & $4.31 \times 10^{-8}$ & $3.00 \times 10^{-9}$ & $6.63 \times 10^{-9}$ & $2.10 \times 10^{-8}$ & $2.00 \times 10^{-7}$ \\
\hline 0.3 & $1.33 \times 10^{-6}$ & $8.40 \times 10^{-8}$ & $3.00 \times 10^{-7}$ & $8.14 \times 10^{-7}$ & $7.01 \times 10^{-6}$ \\
\hline 0.4 & $1.20 \times 10^{-5}$ & $1.61 \times 10^{-6}$ & $5.45 \times 10^{-6}$ & $8.15 \times 10^{-5}$ & $9.21 \times 10^{-5}$ \\
\hline 0.5 & $4.00 \times 10^{-5}$ & $3.00 \times 10^{-5}$ & $6.30 \times 10^{-5}$ & $9.64 \times 10^{-5}$ & $7.00 \times 10^{-4}$ \\
\hline 0.6 & $1.03 \times 10^{-4}$ & $3.55 \times 10^{-4}$ & $5.40 \times 10^{-4}$ & $6.00 \times 10^{-4}$ & $3.30 \times 10^{-3}$ \\
\hline 0.7 & $1.56 \times 10^{-3}$ & $3.00 \times 10^{-3}$ & $4.00 \times 10^{-3}$ & $3.03 \times 10^{-3}$ & $1.20 \times 10^{-2}$ \\
\hline 0.8 & $8.35 \times 10^{-3}$ & $2.00 \times 10^{-2}$ & $2.00 \times 10^{-2}$ & $1.34 \times 10^{-2}$ & $3.34 \times 10^{-2}$ \\
\hline 0.9 & $3.00 \times 10^{-2}$ & $8.00 \times 10^{-2}$ & $9.10 \times 10^{-2}$ & $5.30 \times 10^{-2}$ & $7.00 \times 10^{-2}$ \\
\hline 1. & $6.30 \times 10^{-2}$ & $3.00 \times 10^{-1}$ & $3.63 \times 10^{-1}$ & $2.00 \times 10^{-1}$ & $6.40 \times 10^{-2}$ \\
\hline
\end{tabular}

and nonlinear functions [19]. DJM polynomials for nonlinear term are defined as $[20,21]$

$$
\begin{aligned}
G_{0}(z) & =N\left(y_{0}(z)\right), \\
G_{m}(z) & =N\left(\sum_{n=0}^{m} y_{n}(z)\right)-N\left(\sum_{n=0}^{m-1} y_{n}(z)\right) .
\end{aligned}
$$

The components of $y(z)$, for the series solution, can easily be found by the following recursive process:

$$
\begin{aligned}
y_{0}(z) & =g(z), \\
y_{m+1} & =L\left(y_{m}(z)\right)+G_{m}(z), \quad m=0,1,2,3, \ldots .
\end{aligned}
$$

\section{Analysis of Daftardar Jafari Method}

Consider fourth-order nonlinear boundary value problem

$$
y^{(i v)}(z)=g(z)+h(y(z)),
$$

with boundary conditions

$$
\begin{array}{cc}
y(0)=\alpha_{0}, & y(h)=\alpha_{1}, \\
y^{\prime}(h)=\alpha_{2}, & y^{\prime \prime}(0)=\alpha_{3} .
\end{array}
$$

Using the operator $L=d^{4} / d z^{4},(16)$ can be written as

$$
L(y(z))=g(z)+h(y(z))
$$

Initial conditions are used when the inverse operator $L^{-1}$ is applied on (18) to get

$$
y(z)=\alpha_{0}+\alpha_{1} z+\alpha_{2} \frac{z^{2}}{2 !}+\alpha_{3} \frac{z^{3}}{3 !}+L^{-1} g(z)+L^{-1} h(y(z)) .
$$


In series form (19) can be written as

$$
\begin{aligned}
\sum_{n=0}^{\infty} y_{n}(z)= & \alpha_{0}+\alpha_{1} z+\alpha_{2} \frac{z^{2}}{2 !} \\
& +\alpha_{3} \frac{z^{3}}{3 !}+L^{-1} g(z)+L^{-1}\left(\sum_{n=0}^{\infty} G_{n}(z)\right) .
\end{aligned}
$$

$G_{n}^{\prime} s$ are defined in (14). The components of $y(z)$ can be found as

$$
\begin{aligned}
y_{0}(z) & =\alpha_{0}+\alpha_{1} z+\alpha_{2} \frac{z^{2}}{2 !}+\alpha_{3} \frac{z^{3}}{3 !}, \\
y_{n+1}(z) & =L^{-1}\left(g_{n}(z)+G_{n}(z)\right), \quad n=0,1,2,3, \ldots .
\end{aligned}
$$

For the solution of our problem, we rewrite (12), using the inverse operator $L^{-1}$ which is a fourfold integral with initial conditions (i.e., at $z=0$ ), as

$$
\begin{aligned}
Y(z)= & A z+B \frac{z^{3}}{6}+L^{-1}\left(\operatorname{Me}^{2} Y^{\prime \prime}(z)\right) \\
& -L^{-1}\left(\operatorname{Re} Y(z) \cdot Y^{\prime \prime \prime}(z)\right) .
\end{aligned}
$$

In series form, (22) reduces to

$$
\begin{aligned}
\sum_{n=0}^{\infty} Y_{n}(z)= & A z+B \frac{z^{3}}{6}+L^{-1}\left(\operatorname{Me}^{2} Y^{\prime \prime}(z)\right) \\
& -L^{-1}\left(\operatorname{Re} \sum_{n=0}^{\infty} G_{n}(z)\right) .
\end{aligned}
$$

The last equation gives the components of $Y(z)$. For the nonlinear term $N(Y)=Y(z) \cdot Y^{\prime \prime \prime}(z)$, DJM introduces $G_{n}^{\prime} s$ which are already defined in (14). The recursive process for the components of $Y(z)$ is given by

$$
\begin{aligned}
Y_{0}(z) & =A z+B \frac{z^{3}}{6}, \\
Y_{m+1}(z) & =\operatorname{Me}^{2} L^{-1}\left(Y_{m}^{\prime \prime}(z)\right)-\operatorname{Re} L^{-1}\left(G_{m}(z)\right), \\
& m=0,1,2,3, \ldots .
\end{aligned}
$$

Following the above recursive process, the respective components are obtained as follows:

$$
\begin{aligned}
& Y_{1}(z)=\frac{1}{120}\left(\mathrm{Me}^{2} B-\operatorname{Re} A B\right) z^{5}-\frac{\operatorname{Re} B^{2} z^{7}}{5040} \\
& Y_{2}(z) \\
& =\frac{\left(B M e^{4}-4 B A M e^{2} \operatorname{Re}+3 B A^{2} \operatorname{Re}^{2}\right) z^{7}}{5040} \\
& +\left(-\frac{B^{2} \mathrm{Me}^{2} \operatorname{Re}}{30240}+\frac{B^{2} A \operatorname{Re}^{2}}{22680}\right) z^{9} \\
& +\left(\left(-7 B^{2} \mathrm{Me}^{4} \operatorname{Re}+12 B^{3} \operatorname{Re}^{2}+14 B^{2} A M \mathrm{Me}^{2} \mathrm{Re}^{2}\right.\right. \\
& \left.\left.\quad-7 B^{2} A^{2} \operatorname{Re}^{3}\right) z^{11}\right)(13305600)^{-1}
\end{aligned}
$$

$$
+\frac{\left(B^{3} \mathrm{Me}^{2} \mathrm{Re}^{2}-B^{3} A \mathrm{Re}^{3}\right) z^{13}}{38438400}-\frac{B^{4} \mathrm{Re}^{3} z^{15}}{3962649600}
$$

$Y_{3}(z)$

$$
\begin{aligned}
& =\frac{\left(B M e^{6}-9 B A M e^{4} \operatorname{Re}+23 B A^{2} \mathrm{Me}^{2} \operatorname{Re}^{2}-15 B A^{3} \operatorname{Re}^{3}\right) z^{9}}{362880} \\
& +\frac{\left(-12 B^{2} M e^{4} \operatorname{Re}+61 B^{2} A M e^{2} \operatorname{Re}^{2}-55 B^{2} A^{2} \operatorname{Re}^{3}\right) z^{11}}{9979200} \\
& +\left(\left(-183 B^{2} \mathrm{Me}^{6} \mathrm{Re}+1056 B^{3} \mathrm{Me}^{2} \mathrm{Re}^{2}+1041 B^{2} A M \mathrm{Me}^{4} \mathrm{Re}^{2}\right.\right. \\
& \left.-1684 B^{3} A \operatorname{Re}^{3}-1533 B^{2} A^{2} \mathrm{Me}^{2} \operatorname{Re}^{3}+675 B^{2} A^{3} \operatorname{Re}^{4}\right) \\
& \left.\times(6227020800)^{-1}\right) z^{13} \\
& +\left(\left(-165 B^{2} \mathrm{Me}^{8} \mathrm{Re}+5256 B^{3} \mathrm{Me}^{4} \mathrm{Re}^{2}+1320 B^{2} A M \mathrm{Me}^{6} \mathrm{Re}^{2}\right.\right. \\
& -2988 B^{4} \mathrm{Re}^{3}-13016 B^{3} A M \mathrm{e}^{2} \mathrm{Re}^{3} \\
& -3630 B^{2} A^{2} \mathrm{Me}^{4} \mathrm{Re}^{3}+7760 B^{3} A^{2} \mathrm{Re}^{4} \\
& \left.+3960 B^{2} A^{3} \mathrm{Me}^{2} \mathrm{Re}^{4}-1485 B^{2} A^{4} \mathrm{Re}^{5}\right) \\
& \left.\times(653837184000)^{-1}\right) z^{15}
\end{aligned}
$$

The fifth component $Y_{4}(z)$ is also obtained in the same manner. All the components are truncated to $O\left(z^{17}\right)$. The series solution, using the first five components, is therefore given as

$$
Y(z)=Y_{0}(z)+Y_{1}(z)+Y_{2}(z)+Y_{3}(z)+Y_{4}(z)
$$

This solution involves unknown constants $A, B$. In order to find the values of these unknowns, we use the boundary conditions (i.e., at $z=1$ ). Let us fix the values of $\mathrm{Re}$ and $\mathrm{Me}$ to get the following two equations:

$$
\begin{aligned}
& \left(-\frac{1051 A^{4}}{217945728000}+B\right. \\
& +A^{3}\left(\frac{121223}{108972864000}-\frac{6493 B}{18162144000}+\frac{1357 B^{2}}{25147584000}\right) \\
& +A^{2}\left(-\frac{30503023}{130767436800}+\frac{344689 B}{6671808000}-\frac{2292947 B^{2}}{326918592000}\right. \\
& \left.\quad+\frac{217601 B^{3}}{326918592000}-\frac{11 B^{4}}{792529920}\right) \\
& +A\left(\frac{2331157}{13305600}-\frac{182663 B}{19958400}+\frac{157 B^{2}}{237600}\right. \\
& \left.\left.\quad-\frac{83 B^{3}}{1814400}+\frac{B^{4}}{380160}\right)\right)=1,
\end{aligned}
$$




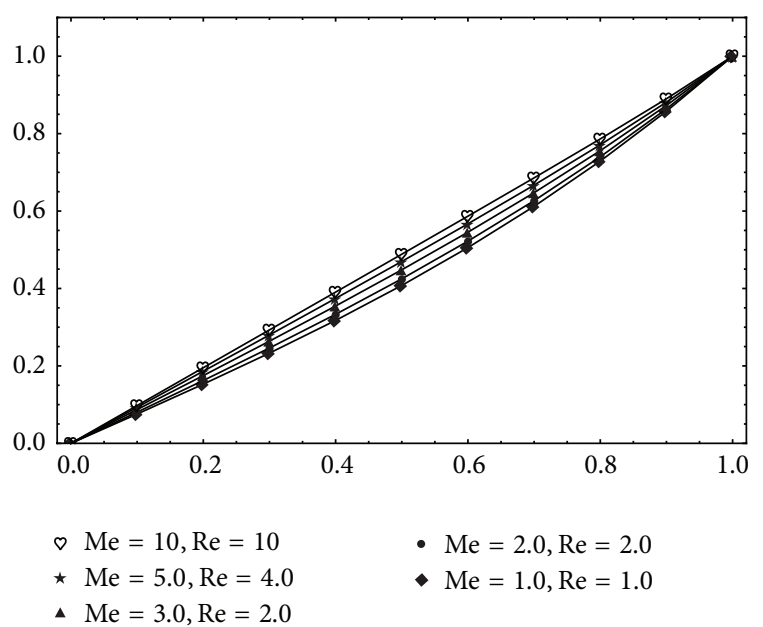

FIGURE 2: Velocity profile for different values of Reynold and Hartmann numbers with $\mathrm{Re} \leq \mathrm{Me}$.

$$
\begin{aligned}
& \left(\frac{1051 A^{4}}{1117670400}+B\right. \\
& +A^{3}\left(-\frac{407}{3386880}+\frac{9103 B}{167650560}-\frac{17641 B^{2}}{1676505600}\right) \\
& +A\left(-\frac{28319}{44800}+\frac{31123 B}{201600}-\frac{631 B^{2}}{25200}+\frac{613 B^{3}}{201600}-\frac{B^{4}}{3840}\right) \\
& +A^{2}\left(\frac{30868891}{3353011200}-\frac{849203 B}{239500800}+\frac{1238579 B^{2}}{1676505600}\right. \\
& \left.\left.\quad-\frac{162581 B^{3}}{1676505600}+\frac{11 B^{4}}{4064256}\right)\right)=0 .
\end{aligned}
$$

The above system of nonlinear equations is solved by using Mathematica software to get

$$
A=1.45539, \quad B=0.754966
$$

and hence solution (26) becomes

$$
\begin{aligned}
Y(z)= & 0.754966 z+0.242565 z^{3}+0.002972 z^{5} \\
& -0.000510 z^{7}+3.91313 \times 10^{-6} z^{9} \\
& +3.15301 \times 10^{-6} z^{11}-1.26484 \times 10^{-7} z^{13} \\
& -2.33163 \times 10^{-8} z^{15} .
\end{aligned}
$$

\section{Numerical Results and Discussion}

The velocity profile of MHD squeezing fluid flow between two parallel plates in porous medium with slip boundary is deduced by using Daftardar Jafari method. All the calculations, tables, and figures are carried out by using Mathematica 7.0. The residuals for different values of slip parameter and Reynolds and Hartmann numbers show the efficiency of the

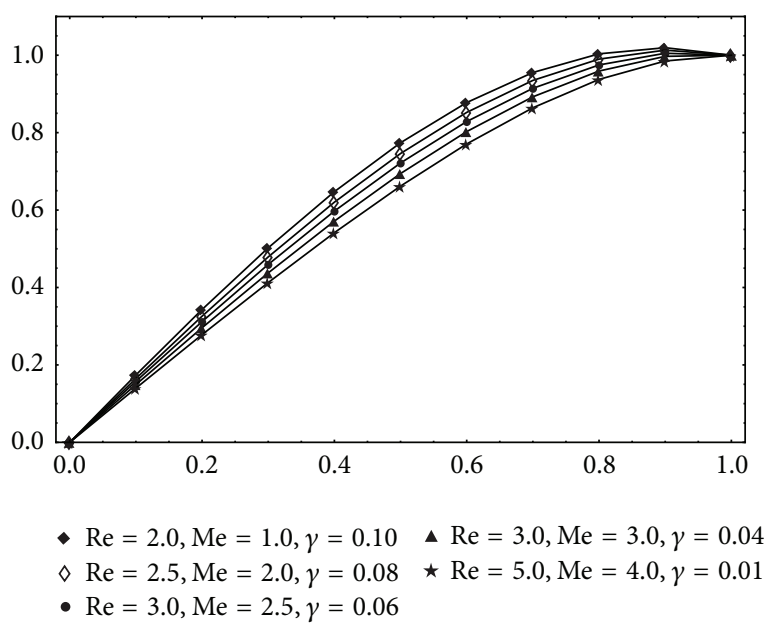

FIGURE 3: Velocity profile for different values of slip parameter and Reynold and Hartmann numbers with $\mathrm{Re} \geq \mathrm{Me}$.

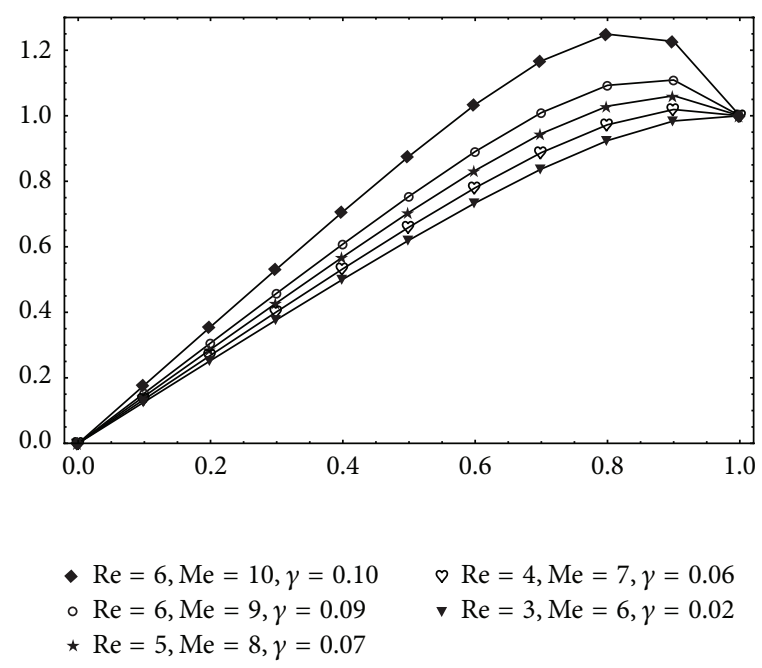

FIGURE 4: Velocity profile for different values of slip parameter and Reynold and Hartmann numbers with $\mathrm{Re}<\mathrm{Me}$.

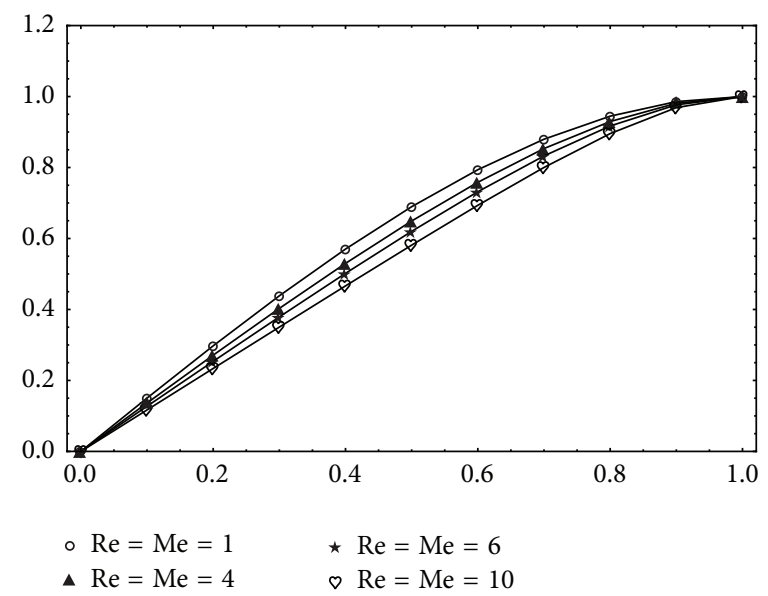

Figure 5: Velocity profile for different values of Reynold and Hartmann numbers with $\operatorname{Re}=\mathrm{Me}$ and $\gamma=0$. 


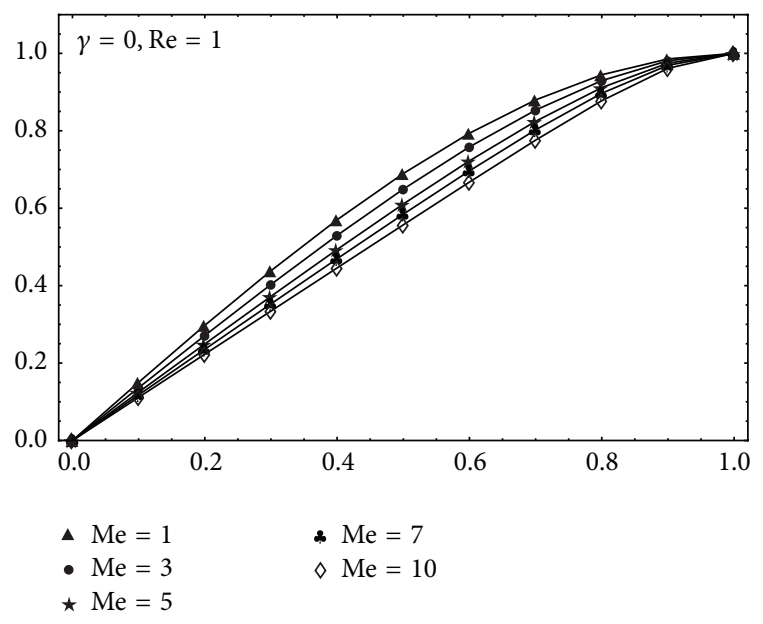

FIGURE 6: Velocity profile for different values of Hartmann number with $\operatorname{Re}=1$ and $\gamma=0$.

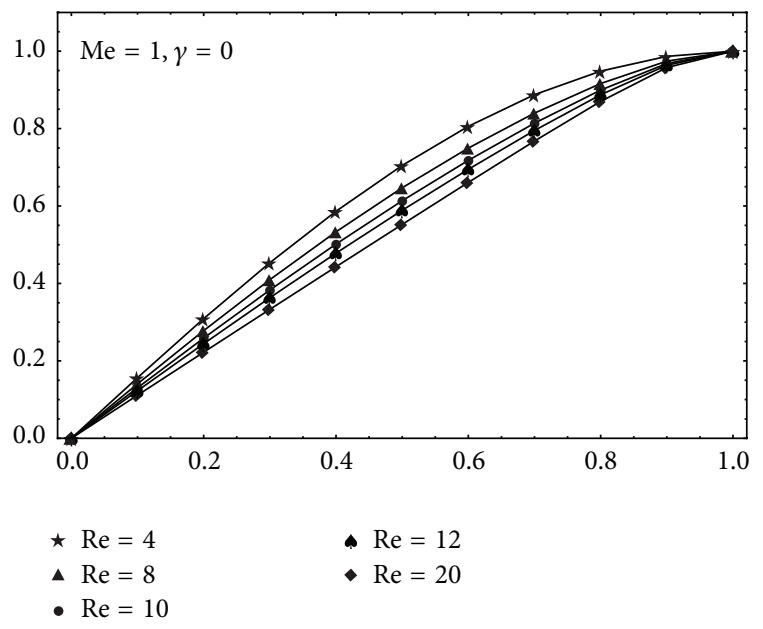

FIGURE 7: Velocity profile for different values of Reynold number with $\mathrm{Me}=1$ and $\gamma=0$.

scheme. This technique requires less computational effort and the convergence of this method to the exact solution is fast enough. The comparison of DJM with other techniques can be found in $[12,21]$. For getting the insight of the problem, we discuss the velocity profile $Y(z)$ of the fluid as follows.

(1) In Figure 2 the influence of Hartmann and Reynolds numbers with constant slip parameter is shown. By decreasing Hartmann number with $\mathrm{Me} \geq \mathrm{Re}$, we observe from Figure 2 that the velocity of the fluid decreases.

(2) The influence of Reynolds and Hartmann numbers for different values of slip parameter is shown in Figure 3. It can be seen in Figure 3 that by increasing Reynolds and Hartmann numbers with $\mathrm{Re} \geq \mathrm{Me}$ and decreasing the slip parameter $\gamma$, the velocity decreases. The same effect on the velocity profile is shown in Figure 4 by decreasing Reynolds and Hartmann numbers with $\mathrm{Re}<\mathrm{Me}$.
(3) Figures 5, 6, and 7 are special cases when the slip parameter $\gamma$ vanishes. From Figure 5 we conclude that the velocity increases with equal increase in $\mathrm{Me}$ and Re. In the same way the increase in velocity is observed in Figures 6 and 7 while increasing Me and Re, keeping Reynolds and Hartmann numbers fixed, respectively.

\section{Conflict of Interests}

The authors declare that there is no conflict of interests regarding the publication of this paper.

\section{References}

[1] T. C. Papanastasiou, G. C. Georgiou, and A. N. Alexandrou, Viscous Fluid Flow, CRC Press, New York, NY, USA, 1994.

[2] M. J. Stefan, "Versuch Uber die scheinbare adhesion," Akademie der Wissenschaften, vol. 69, article 713, 1874.

[3] X. J. Ran, Q. Y. Zhu, and Y. Li, "An explicit series solution of the squeezing ow between two innite parallel plates," Communications in Nonlinear Science and Numerical Simulation, vol. 8, no. 2, pp. 179-184, 2007.

[4] R. J. Grimm, "Squeezing flows of Newtonian liquid films an analysis including fluid inertia," Applied Scientific Research, vol. 32, no. 2, pp. 149-166, 1976.

[5] W. F. Hughes and R. A. Elco, "Magnetohydrodynamic lubrication flow between parallel rotating disks," Journal of Fluid Mechanics, vol. 13, pp. 21-32, 1962.

[6] S. Kamiyama, "Inertia effects in MHD hydrostatic thrust bearing," Journal of Tribology, vol. 91, no. 4, pp. 589-596, 1969.

[7] E. A. Hamza, "The magnetohydrodynamic squeeze film," Journal of Tribology, vol. 110, no. 2, pp. 375-377, 1988.

[8] S. Bhattacharyya and A. Pal, "Unsteady MHD squeezing flow between two parallel rotating discs," Mechanics Research Communications, vol. 24, no. 6, pp. 615-623, 1997.

[9] C. L. M. H. Navier, "Sur les lois de l'équilibre et dumouvement des corps solides elastiques," Bulletin des Sciences par la Societe Philomatique de Paris, pp. 177-181, 1823.

[10] C. L. Roux, "Existence and uniqueness of the flow of secondgrade fluids with slip boundary conditions," Archive for Rational Mechanics and Analysis, vol. 148, no. 4, pp. 309-356, 1999.

[11] L. J. Rhooades, R. Resnic, T. O’Bradovich, and S. Stegman, "Abrasiveflow machining of cylinder heads and its positive effects on performance and cost characteristics," Tech. Rep., SAE International, Dearborn, Mich, USA, 1996.

[12] I. Ullah, H. Khan, and M. T. Rahim, "Approximation of first grade MHD squeezing fluid flow with slip boundary condition using DTM and OHAM," Mathematical Problems in Engineering, vol. 2013, Article ID 816262, 9 pages, 2013.

[13] T. Hayat and S. Abelman, "A numerical study of the influence of slip boundary condition on rotating flow," International Journal of Computational Fluid Dynamics, vol. 21, no. 1, pp. 21-27, 2007.

[14] S. Abelman, E. Momoniat, and T. Hayat, "Steady MHD flow of a third grade fluid in a rotating frame and porous space," Nonlinear Analysis: Real World Applications, vol. 10, no. 6, pp. 3322-3328, 2009.

[15] A. Ebaid, "Effects of magnetic field and wall slip conditions on the peristaltic transport of a Newtonian fluid in an asymmetric channel," Physics Letters A: General, Atomic and Solid State Physics, vol. 372, no. 24, pp. 4493-4499, 2008. 
[16] V. Daftardar-Gejji and H. Jafari, "An iterative method for solving nonlinear functional equations," Journal of Mathematical Analysis and Applications, vol. 316, no. 2, pp. 753-763, 2006.

[17] S. Bhalekar and V. Daftardar-Gejji, "New iterative method: application to partial differential equations," Applied Mathematics and Computation, vol. 203, no. 2, pp. 778-783, 2008.

[18] I. Ullah, H. Khan, and M. T. Rahim, "Numerical solutions of higher order nonlinear boundary value problems by new iterative method," Applied Mathematical Sciences, vol. 7, no. 4952, pp. 2429-2439, 2013.

[19] V. Daftardar-Gejji and S. Bhalekar, "Solving fractional boundary value problems with Dirichlet boundary conditions using a new iterative method," Computers \& Mathematics with Applications, vol. 59, no. 5, pp. 1801-1809, 2010.

[20] H. Khan, S. Islam, J. Ali, and I. Ali Shah, "Comparison of different analytic solutions to axisymmetric squeezing fluid flow between two infinite parallel plates with slip boundary conditions," Abstract and Applied Analysis, vol. 2012, Article ID 835268, 18 pages, 2012.

[21] I. ullah, H. Khan, and M. T. Rahim, "Numerical solutions of fifth and sixth order nonlinear boundary value problems by Daftardar Jafari method," Journal of Computational Engineering, vol. 2014, Article ID 286039, 8 pages, 2014. 


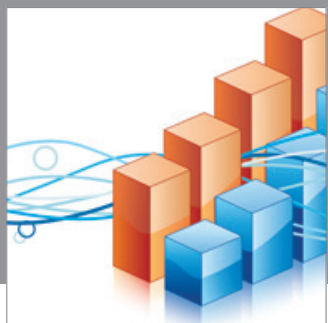

Advances in

Operations Research

mansans

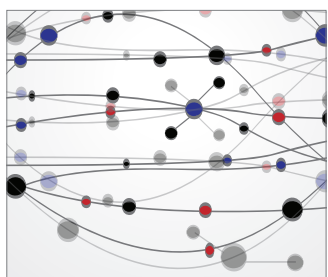

The Scientific World Journal
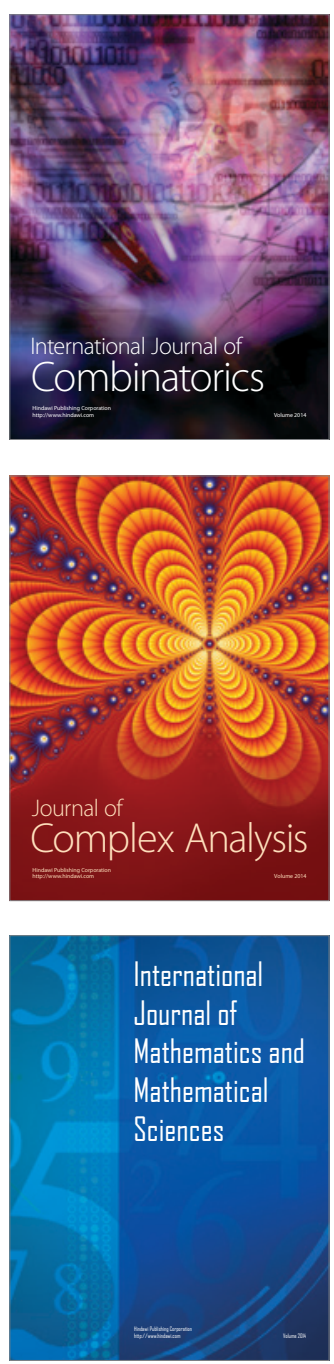
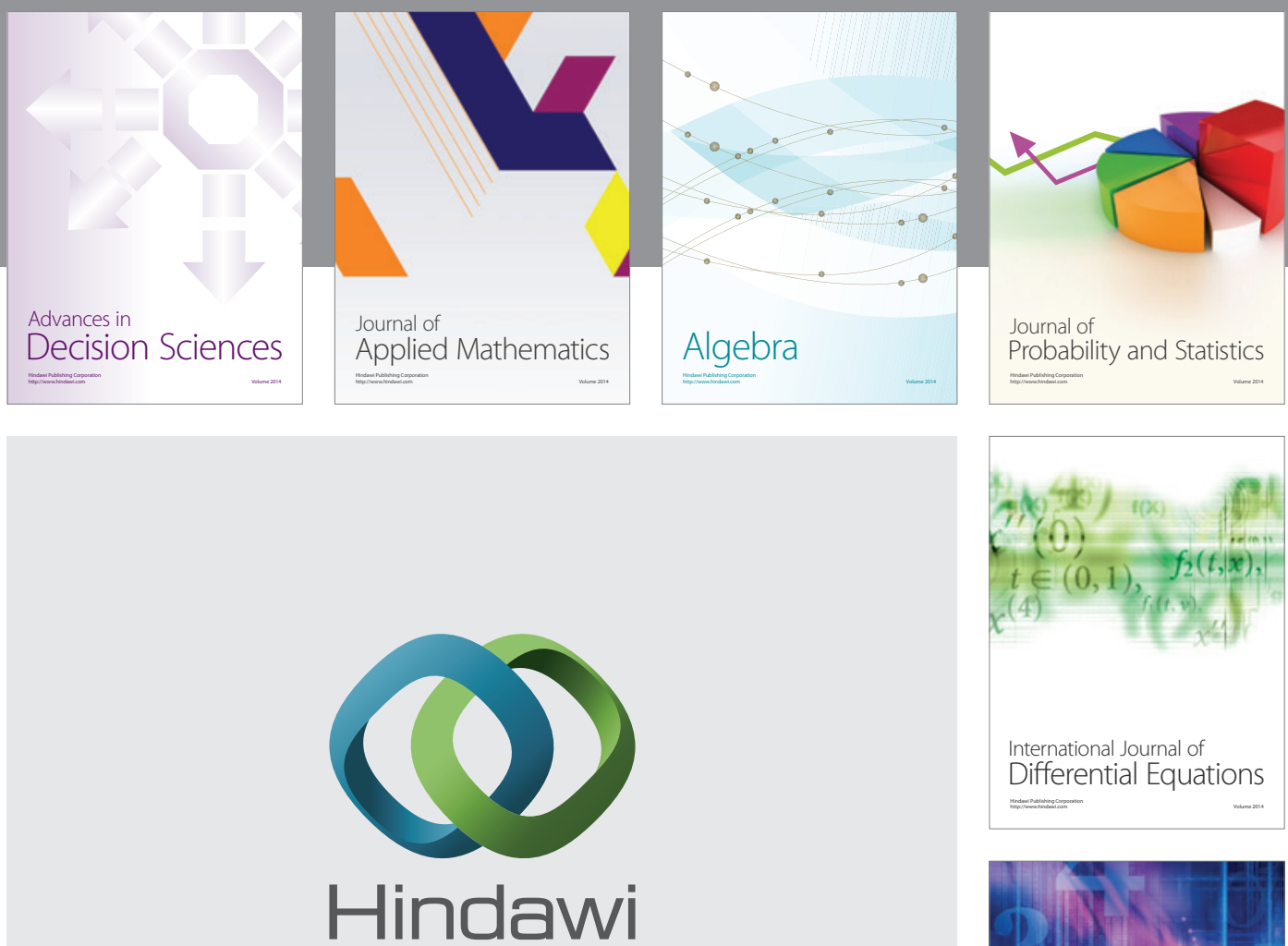

Submit your manuscripts at http://www.hindawi.com
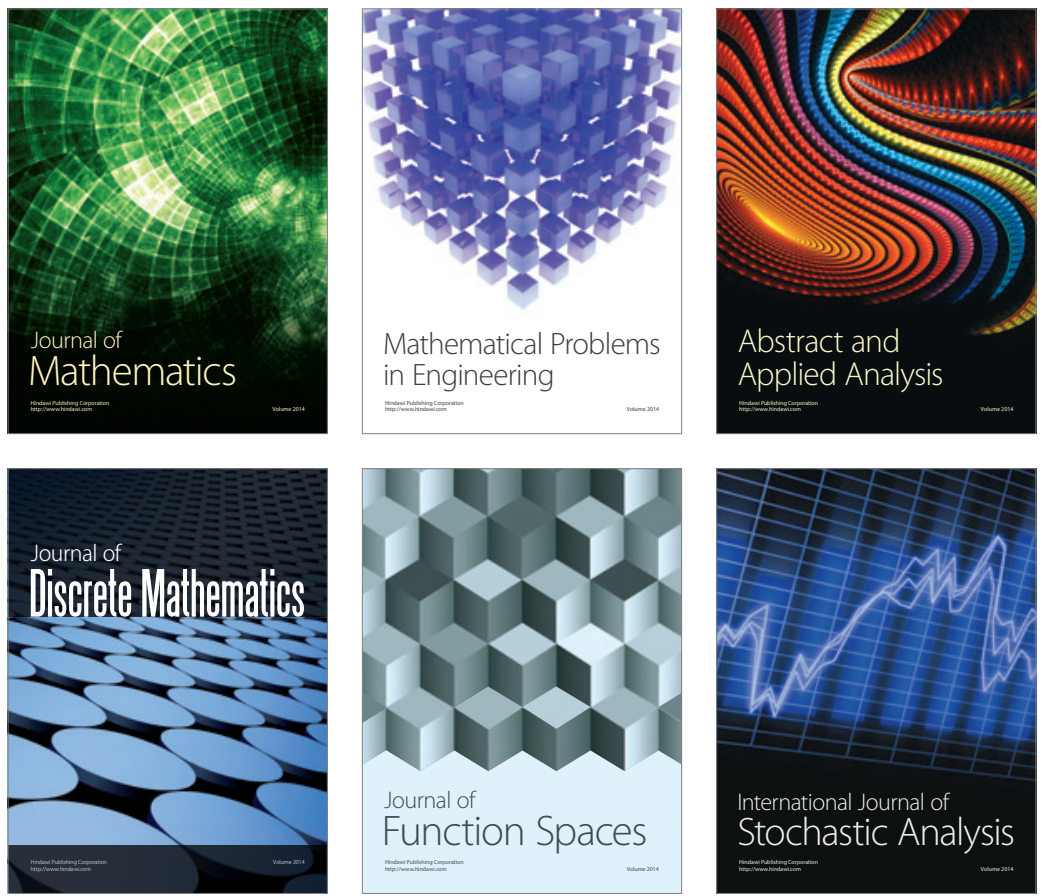

Journal of

Function Spaces

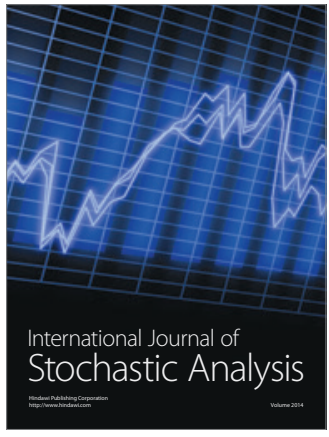

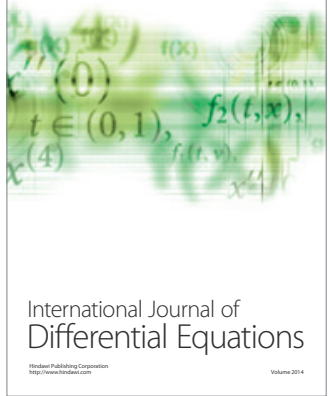
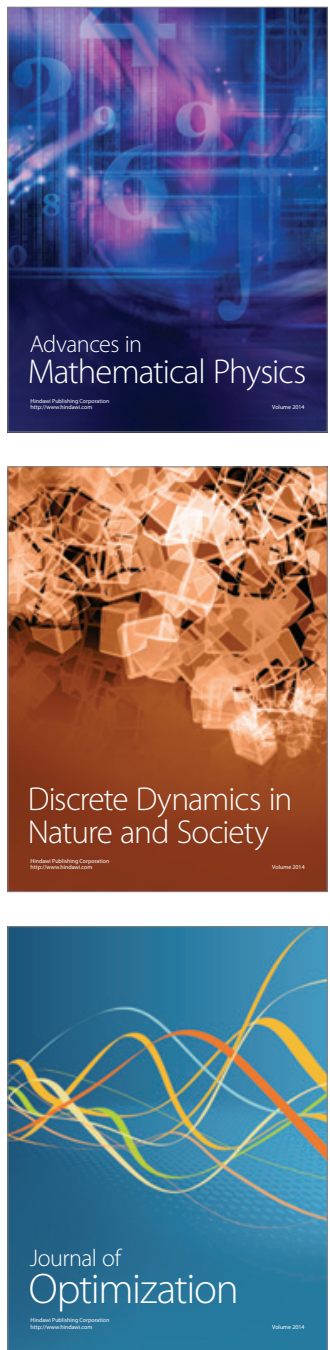